\title{
Reduced albedo on early Mars does not solve the climate paradox under a faint young Sun
}

\author{
A. G. Fairén ${ }^{1}$, J. D. Haqq-Misra ${ }^{2}$, and C. P. McKay ${ }^{1}$ \\ ${ }^{1}$ Space Science Division, NASA Ames Research Center, Moffett Field, CA 94035, USA \\ e-mail: alberto.g.fairen@nasa.gov \\ 2 Blue Marble Space Institute of Science, PO Box 85561, Seattle, WA 98145, USA \\ e-mail: jacob@bmsis.org
}

Received 27 November 2011 / Accepted 7 February 2012

\section{ABSTRACT}

\begin{abstract}
Context. The presence of liquid water on the surface of early Mars and Earth is difficult to reconcile with the reduced solar luminosity at $3.8 \mathrm{Ga}$. and before, which would have imposed mean temperatures below freezing all over both planets. For the case of Earth, it has been recently suggested the hypothesis that less continental area and limited cloudiness during the Archaean may have reduced planetary albedo and thereby increased surface warming by sunlight.

Aims. Here we analyze whether this novel solution explaining warming conditions on the early Earth could be applied to early Mars. Methods. We use an energy balance climate model in our calculations.

Results. Our results show that early Mars could have been kept warm as long as there was a nearly global ocean and relatively sparse cloud coverage. This result is internally inconsistent, and also incompatible with most of the observed geological evidence.

Conclusions. Reduced albedo is not a suitable solution for the faint young Sun problem in the case of early Mars. The combination of climatic and geochemical models is essential for understanding the stability of liquid water during the Noachian.
\end{abstract}

Key words. planets and satellites: individual: Mars - planets and satellites: atmospheres - planets and satellites: surfaces

\section{Introduction: The problem of a "warm" early Mars}

Geologic evidence of flowing and ponding liquid water on the surface of ancient Mars appears abundantly across most of the Martian landscape (Baker et al. 1991; Parker et al. 1993; Head et al. 1998; Clifford \& Parker 2001; Fairén et al. 2003; Malin \& Edgett 2003; Squyres et al. 2004; Irwin et al. 2005; Pain et al. 2007; Di Achille \& Hynek 2010), indicating that liquid water has been present in variable amounts and for long periods of time on and/or near the surface at different moments of Mars' history. Early Mars appears to have been "wet". However, the presence of liquid water on the surface of early Mars is difficult to reconcile with the reduced solar luminosity at $3.8 \mathrm{Ga}$. and before (with a relative solar constant $S / S_{0} \approx 0.75$ so that the Sun was about 25\% fainter than today; Gough 1981; Bahcall et al. 2001), which would have imposed mean temperatures below freezing all over the planet. Atmospheric greenhouse gases (Sagan \& Mullen 1972; Squyres \& Kasting 1994; Sagan \& Chyba 1997; Halevy et al. 2007) and carbon dioxide ice clouds in the upper troposphere (Forget \& Pierrehumbert 1997) are suspected to explain some of this discrepancy, but these solutions have been probed to face numerous problems (Kuhn \& Atreya 1979; Squyres \& Kasting 1994; Kasting 1997; Haberle 1998; Johnson et al. 2008; Grott et al. 2011), as discussed elsewhere (Fairén et al. 2009; Fairén 2010). So, was early Mars "warm"?

This approach to understanding the Martian climate when the young Sun was fainter has also been applied to early Earth, because liquid water appears to have equally been present early on our planet. However, if we assume that the climate during the Archaean was relatively similar to present-day Earth, then global temperature would fall below the freezing point of water-just as is postulated for early Mars. The same solutions, namely additional atmospheric greenhouse effects from high partial pressures of $\mathrm{CO}_{2}, \mathrm{NH}_{4}, \mathrm{CH}_{4}$ and $\mathrm{C}_{2} \mathrm{H}_{6}$, have been suggested as an explanation to the early Earth's problem (Kiehl \& Dickinson 1987; Kasting 1993; Sagan \& Chyba 1997; Catling et al. 2001; von Paris et al. 2008; Haqq-Misra et al. 2008). An alternative solution has been recently proposed (Rosing et al. 2010) that a lower albedo on the Earth, owing to considerably less continental area and to the lack of biologically induced cloud condensation nuclei, made an important contribution to moderating surface temperature in the Archaean eon. The lower albedo of the early Earth would have provided environmental conditions above the freezing point of water, thus alleviating the need for extreme greenhouse-gas concentrations to satisfy the faint early Sun paradox. This alternative solution for the early Earth has already been debated elsewhere (Dauphas \& Kasting 2011; Goldblatt \& Zahnle 2011; Rosing et al. 2011), and it is not our intention here to ponder the accuracy of the Rosing et al. (2010) model for the early Earth. The only aim of this paper is to analyze whether this novel solution for the early Earth could be applied to early Mars, where the issue is even more problematic because of the greater distance from the Sun. Although Rosing et al. (2010) do not suggest an implication of their work for early Mars, we wanted to explore the relevance of this new model for the other planet in the solar system where the same problem has been highlighted, therefore constraining the applicability of the proposed model for planets other than Earth.

\section{Methods: energy balance model for early Mars}

In our calculations, we use an energy balance climate model (EBM) derived from a previous study (Williams \& Kasting 1997). In this model, absorption of thermal infrared radiation at 
each latitude band is parameterized using results from an existing radiative-convective model (Kasting 1991), and heat transport between latitudinal bands is modeled as diffusion. At the mean Earth-Sun orbital distance of $a_{0}=1.0 \mathrm{AU}$, the solar flux $q_{0}$ is $1360 \mathrm{~W} \mathrm{~m}^{-2}$. The diurnally averaged incident solar flux $S$ for a planet with an orbital semimajor axis $a$ can be expressed by Eq. (A.8) in Williams \& Kasting (1997):

$S=\frac{q_{0}}{\pi}\left(\frac{a_{0}}{a}\right)^{2}(H \sin \theta \sin \delta+\cos \theta \cos \delta \sin H)$,

where $\theta$ is latitude and $\delta$ is solar declination. The radian halfday length $H$ is given by the relation $\cos H=-\tan \theta \tan \delta$, for $0<H<\pi$.

The albedo of an unfrozen ocean is a function of the solar zenith angle $Z$ and can range from 0 to 1.0. The fraction of incoming solar radiation reflected by a smooth oceanic surface was tabulated from Fresnel reflectance formulas for water (Kondrat'ev 1969) and is notated functionally as $\mathcal{F}(Z)$. From $263 \mathrm{~K}$ to $273 \mathrm{~K}$, the fraction of oceanic ice cover $f_{i}$ is parameterized from data (Thomson \& Barron 1981) as a function of surface temperature $T$ so that $f_{i}=1-\exp [(T-273 \mathrm{~K}) / 10 \mathrm{~K}]$. A completely frozen ocean has an albedo of 0.663 (Caldeira \& Kasting 1992), so the ocean albedo $a_{\mathrm{o}}$ is expressed as:

$$
a_{\mathrm{o}}= \begin{cases}\mathcal{F}(Z) & \text { for } T \geq 273 \mathrm{~K} \\ \mathcal{F}(Z)\left(1-f_{i}\right)+0.663 f_{i} & \text { for } 263 \mathrm{~K}<T<273 \mathrm{~K} \\ 0.663 & \text { for } T \leq 263 \mathrm{~K}\end{cases}
$$

Unfrozen land is assigned a characteristic surface albedo of 0.20 (Kondrat'ev 1969), while fractional snow cover occurs on land below freezing. The expression for the land albedo $a_{1}$ is

$$
a_{1}= \begin{cases}0.20 & \text { for } T \geq 273 \mathrm{~K} \\ 0.663 \mathcal{L}+0.20(1-\mathcal{L}) & \text { for } T<273 \mathrm{~K}\end{cases}
$$

where $\mathcal{L}$ is the fraction of land on which snowfall occurs. The fraction of the surface covered by water clouds is described by the parameter $f_{\mathrm{c}}$. Cloud albedo $a_{\mathrm{c}}$ is parameterized as a linear function of $Z$ as in Eq. (A.14) of Williams \& Kasting (1997): $a_{\mathrm{c}}=\alpha+\beta Z$. The values $\alpha=-0.078$ and $\beta=0.65$ allow the model to reproduce the latitudinal albedo distribution of Earth (Jacobowitz et al. 1979). A wet early Mars would arguably be more like present-day Earth than present-day Mars, so we have selected our model parameters to reflect such a climate.

The fraction of the surface covered by water clouds is described by the parameter $f_{\mathrm{c}}$ so that we can account for the effect of partial cloudiness by a reduction in cloud albedo. Clouds only affect albedo in this model, so the surface steadily cools as cloud cover increases until the planetary albedo becomes large enough to initiate global glaciation. Likewise, an increase in snowfall corresponds to a larger planetary albedo so that greater snow coverage causes the surface to glaciate at lower values of cloud coverage. We can therefore think of cloud fraction $f_{\mathrm{c}}$ as a tunable parameter that reflects the contribution of clouds to planetary albedo. The question of the biologically induced cloud condensation nuclei (included in Rosing et al. 2010) is not considered here, as we assume a lifeless planet during the Noachian eon for the purposes of this work.

We choose the maximum value between ice albedo and cloud albedo below the freezing point of water in order to keep the presence of clouds from darkening a bright ice-covered surface. Letting $f_{\mathrm{o}}$ be the fraction of the surface covered by ocean, the total zonally averaged surface albedo as can now be expressed as:

$a_{\mathrm{s}}=\frac{1}{2} \begin{cases}\left(1-f_{\mathrm{o}}\right) a_{\mathrm{l}}+f_{\mathrm{o}} a_{\mathrm{o}}+a_{\mathrm{c}} & \text { for } T \geq 273 \mathrm{~K} \\ \max \left[\left(1-f_{\mathrm{o}}\right) a_{1}+f_{\mathrm{o}} a_{\mathrm{o}}, a_{\mathrm{c}}\right] & \text { for } T<273 \mathrm{~K} .\end{cases}$

The top-of-atmosphere (TOA) albedo $\mathcal{A}$ follows a second-order polynomial parametrization of the radiative-convective model described in Kasting (1991) and depends on $T, a_{\mathrm{s}}, Z$, and carbon dioxide partial pressure $\left(p \mathrm{CO}_{2}\right)$. Given in Eqs. (A.9) and (A.10) of Williams \& Kasting (1997), this parametrization is valid for $190 \mathrm{~K} \leq T \leq 360 \mathrm{~K}, 10^{-5}$ bar $\leq p \mathrm{CO}_{2} \leq 10$ bar, and $0 \leq a_{\mathrm{s}} \leq 1$ over all solar zenith angles. In a likewise manner, the outgoing infrared radiation flux $\mathcal{I}$ is parameterized as a third-order polynomial function of $T$ and $p \mathrm{CO}_{2}$ (Eq. (A.18) in Williams \& Kasting 1997) and is valid over the same range of temperatures and pressures as TOA albedo.

The effective zonal heat capacity of atmosphere over land $C_{1}$ is $5.25 \times 10^{6} \mathrm{~J} \mathrm{~m}^{-2} \mathrm{~K}^{-1}$ (North et al. 1983). Over the ocean, the heat capacity $C_{\mathrm{o}}=40 C_{1}$ for $T \geq 273 \mathrm{~K}$, corresponding to a wind-mixed $50 \mathrm{~m}$ ocean layer, while for $T<273 \mathrm{~K}$ the heat capacity over ice is $C_{i}=2 C_{1}$ (Williams \& Kasting 1997). This gives an expression for the zonally averaged effective heat capacity $C$ :

$C=\left(1-f_{i}\right) C_{1}+f_{\mathrm{o}}\left[\left(1-f_{i}\right) C_{\mathrm{o}}+f_{i} C_{i}\right]$.

Latitudinal dynamical energy transport is encapsulated in the diffusion parameter $D$. In order to apply this model to a range of planetary atmospheres, the diffusion parameter is expressed following Williams \& Kasting (1997) as

$\left(\frac{D}{D_{0}}\right)=\left(\frac{p}{p_{0}}\right)\left(\frac{c_{p}}{c_{p_{0}}}\right)\left(\frac{m_{0}}{m}\right)^{2}\left(\frac{\Omega_{0}}{\Omega}\right)^{2}$,

where $p$ is atmospheric pressure, $c_{p}$ is specific heat capacity, $m$ is the mean molecular weight, and $\Omega$ is the planetary rotation rate. The subscript 0 refers to present Earth, with $p_{0}=1 \mathrm{bar}$, $c_{p_{0}}=1000 \mathrm{~J} \mathrm{~kg}^{-1} \mathrm{~K}^{-1}, m_{0}=28.0 \mathrm{~g} \mathrm{~mol}^{-1}$, and $\Omega_{0}=7.27 \times$ $10^{-5} \mathrm{rad} \mathrm{s}^{-1}$. The parameter $D_{0}$ is adjusted to $0.58 \mathrm{~W} \mathrm{~m}^{-2} \mathrm{~K}^{-1}$ in order to match the latitudinal temperature gradient on Earth.

To construct the energy balance equation, the amount of absorbed incoming solar radiation must be balanced by transport processes and infrared emission to space. Let $x=\sin \theta$ and $t$ be the time. The radiative and dynamic energy balance is then

$S(1-\mathcal{A})=\mathcal{I}-\frac{\partial}{\partial x} D\left(1-x^{2}\right) \frac{\partial T(x, t)}{\partial x}+C \frac{\partial T(x, t)}{\partial t}$.

From left to right, the terms in Eq. (7) represent absorbed incoming solar radiation, emitted outgoing infrared radiation, latitudinal energy transport, and thermal inertia of the ocean and atmosphere (cf., Eq. (53) in North et al. 1981 or Eq. (1) in Williams \& Kasting 1997).

To solve the energy balance in Eq. (7), Mars is initialized with a temperature profile of $T=288 \mathrm{~K}$ at all latitudes and stepped through its orbit by increments of $\Delta t$. For each $\Delta t$, the solar declination specifies a value of solar insolation in Eq. (1), while the TOA albedo $\mathcal{A}$ and outgoing infrared flux $\mathcal{I}$ depend on surface temperature. Dynamical energy transport is calculated across all latitude belts, and the value of the pressure-dependent diffusion coefficient $D$ is updated according to Eq. (6). Surface temperatures are then updated, and the numerical integration of Eq. (7) continues until reaching a statistically steady state.

In our energy balance model, warming is maximized for $0 \%$ cloud coverage, because clouds are reflective in this model and 
contribute to planetary albedo but do not directly provide any warming, so we cannot account for any warming that could be provided from infrared absorption or scattering by clouds (Forget \& Pierrehumbert 1997). Also, these calculations are not at all sensitive to the effect of snowfall on land because land surface area is much smaller than ocean coverage, so we do not include this in our graphic results.

The radiative transfer scheme described above was parameterized for computational efficiency so that a wide parameter space of climate variables can be calculated quickly. This parameterization is based on the radiative convective model of Kasting (1991) and applies across a range of $\mathrm{CO}_{2}$ and $\mathrm{H}_{2} \mathrm{O}$ atmospheres. These type of radiative-convective models are limited in their ability to produce the effects of fractional cloud cover; in the case of the Kasting (1991) model, the only effect of clouds is to increase the surface albedo of Earth. Surface albedo in this model is therefore considered as a tunable parameter that is adjusted in order to allow the model to reproduce an Earth-like climate under present-day conditions. This approach, as well as possible alternatives, have been discussed (Goldblatt \& Zahnle 2010; Rondanelli \& Lindzen 2010), but three dimensional general circulation models are ultimately required to properly constrain the effects of partial cloud cover.

Additionally, recent updates to the thermal absorption of the Kasting (1991) model are absent in our parameterization. Some of the improvements to this radiative convective model include an optimized vertical resolution, which can resolve up to ten degrees of additional warming compared with previous calculations (Tian et al. 2010). However, improvements to the treatment of collision-induced absorption by carbon dioxide molecules have shown a decrease in the amount of warming possible by atmospheric $\mathrm{CO}_{2}$ (Wordsworth et al. 2010). In fact, the calculations by Wordsworth et al. (2010) find that a 3 bar $\mathrm{CO}_{2}$ atmosphere may be as much as $10 \mathrm{~K}$ cooler in their improved treatment of collision-induced absorption than the earlier model by Kasting et al. (1984). This decrease in the expected amount of warming from atmospheric $\mathrm{CO}_{2}$ outweighs any of the other improvements made to these radiative transfer calculations, so our calculations here can be considered as an overestimation of the greenhouse effect realizable by a dense $\mathrm{CO}_{2}-\mathrm{H}_{2} \mathrm{O}$ atmosphere. In general, we can use our EBM as an optimistic upper limit, with a margin of error, to illustrate a range of plausible atmospheres for early Mars.

\section{Modeling: early Martian climate}

We first apply our model to early Earth in order to explore the degree to which a lower albedo could have kept the planet warm. The low-albedo hypothesis (Rosing et al. 2010) suggests that less continental area and limited cloudiness during the Archaean may have reduced planetary albedo and thereby increased surface warming by sunlight. Additionally, calculations for this hypothesis place an upper limit of 1600 ppmv for atmospheric $\mathrm{CO}_{2}$ and 800 ppmv for atmospheric $\mathrm{CH}_{4}$, which limits the amount of warming provided by greenhouse gases. Radiative forcing in our EBM is parameterized as a function of $\mathrm{CO}_{2}$ only, so we cannot calculate the contribution from $\mathrm{CH}_{4}$ directly; however, we can use existing radiative-convective equilibrium model calculations for the Archaean Earth (Haqq-Misra et al. 2008), which show that the total amount of warming provided by $1600 \mathrm{ppmv}$

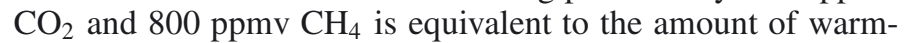
ing provided by about 10000 ppmv of atmospheric carbon dioxide. We use this value for the partial pressure of $\mathrm{CO}_{2}$ for our EBM calculations in Fig. 1, which shows the mean annual

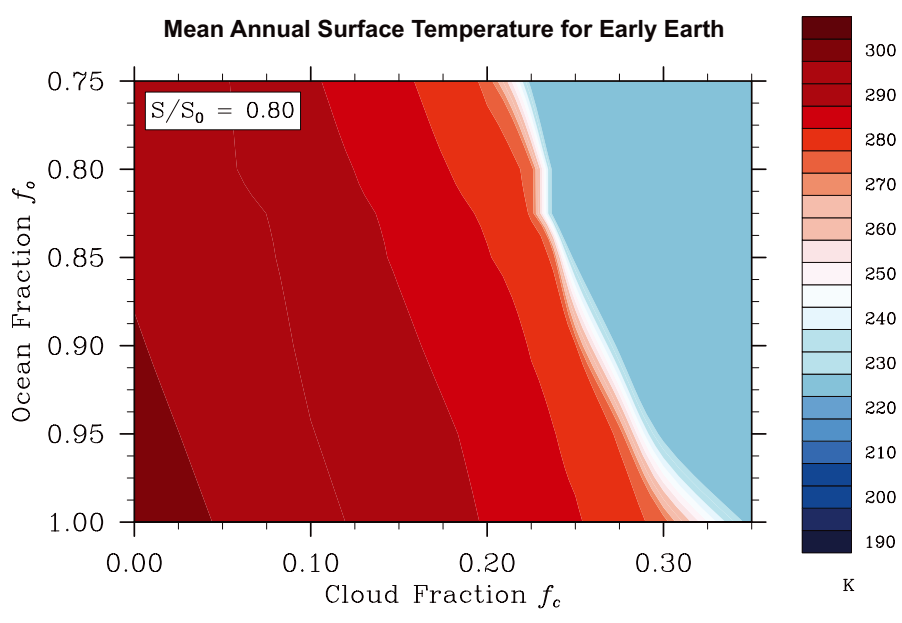

Fig. 1. Mean annual surface temperature for early Earth. Calculated at a relative solar constant $S / S_{0}=0.80$, and as a function of cloud fraction and ocean fraction. The amount of warming from greenhouse gases in these calculations is equivalent to the warming at the maximum concentrations of atmospheric $\mathrm{CO}_{2}$ and $\mathrm{CH}_{4}$ described by Rosing et al. (2010).

surface temperature for the Archaean Earth $\left(S / S_{0}=0.80\right)$ as a function of cloud fraction $f_{\mathrm{c}}$ and ocean fraction $f_{\mathrm{o}}$. Our calculations show that the low-albedo hypothesis (Rosing et al. 2010) is tenable: larger ocean coverage provides for warmer climate, while reduced cloud cover also allows the surface to warm above the freezing point of water. Note that the faint young Sun paradox is illustrated here by the fact that conditions analogous to present-day Earth $\left(f_{\mathrm{c}}=0.5\right.$ and $\left.f_{\mathrm{o}}=0.75\right)$ correspond to global glacial conditions in Fig. 1; a warm early Earth seems to require either a lower planetary albedo or additional greenhouse gas forcing. We should also note that our EBM requires a cloud fraction of $\leq 30 \%$ to produce an above-freezing climate for the maximum greenhouse warming described in the low-albedo hypothesis (Rosing et al. 2010). Our cloud scheme is admittedly far too simplistic to be useful as an accurate predictor of fractional cover, but that estimate is beyond the scope of this work, and our calculations should only be taken as a proof of concept to demonstrate that the low-albedo hypothesis is possible and reproducible with our model.

We now turn our attention to early Mars, where the combination of a faint young Sun and a greater orbital distance yield a relative solar constant $S / S_{0} \approx 0.33$. We maximize the greenhouse warming in our model by using a 3 bar $\mathrm{CO}_{2}$ atmosphere, which corresponds to the maximum amount of warming that can be realized by a one-dimensional model (Kasting 1991; Tian et al. 2010). So far climate models have been unable to explain warm conditions on early Mars in the presence of a faint young sun (Haberle 1998), and our EBM is no exception. Part of the reason for this failure to find a climate that is consistent with the geological evidence on Mars is that one-dimensional climate models are unable to explicitly represent processes such as fractional cloud cover or heat transport by atmospheric dynamics, which could supplement the warming provided by radiative transfer. Three-dimensional processes are ultimately necessary to fully understand the stability of liquid water in a planetary surface, but for our analysis in this paper, we simply choose a reasonable partial pressure for $\mathrm{CO}_{2}$ that corresponds to an optimal scenario for a warm early Mars. If in fact early Mars had significantly less greenhouse forcing than is equivalent to the 3 bar atmosphere we are considering here, then our calculations below can be considered as an upper limit. 


\section{Results and discussion: a waterworld as the only model solution}

We have calculated surface temperatures for early Mars by assuming a northern "superocean" with a varying total areal extension: all the land is surrounding the South Pole, and the ocean is north of this. We also consider the effect of Mars' chaotic obliquity over time and present our calculations over the range of obliquities likely experienced by Mars (Touma \& Wisdom 1993; Laskar \& Robutel 1993; Laskar et al. 2004), taking into consideration that the range of obliquities experienced by Mars early in its history is not yet completely understood, and long-term stable obliquities of $0^{\circ}$, which we have included in our calculations, might be possible (Brasser \& Walsh 2011). Using these parameters for early Mars, we actually do get above-freezing temperatures for our calculations. As described in Fig. 2, warm conditions are attained at an ocean fraction of $f_{\mathrm{o}} \geq 0.95$ and a cloud fraction $f_{\mathrm{c}} \leq 0.05$. The climate system in our EBM is highly bistable to both cloud and ocean fraction, while the range of parameter space that allows for a warm climate is relatively small compared to early Earth (Fig. 1). Even so, these calculations indicate that a low albedo could help explain warm conditions on early Mars under the influence of a faint young Sun, as long as there was a nearly global ocean and relatively sparse cloud coverage.

Therefore, when applied to early Mars, the low-albedo hypothesis requires that Mars should have been a "cloudless waterworld" during the Noachian to explain the presence of surface liquid water. It is somewhat difficult to believe that a $\geq 95 \%$ ocean world would really be almost cloud-free, because a nearly global ocean and relatively sparse cloud coverage are somewhat contradictory requirements. After all, the atmosphere above a waterworld would accumulate moisture from evaporation, at least in the unfrozen tropics, so that moist surface-based convection can inject cloud condensation nuclei into the troposphere and lift water vapor aloft. A waterworld, then, should be at least somewhat conducive to cloud formation. Our result is also inconsistent with most of the observed geological evidence, which does not provide any indication of a globally inundated Martian surface during the Noachian. The geological record may only support an ocean covering a maximum of $1 / 3$ of the planet (Parker et al. 1993; Head et al. 1998), and even this possibility is controversial. Thus, it may be physically unreasonable to explain the problem of the faint young Sun on early Mars in terms of a low planetary albedo.

\section{Conclusions: faint young Sun paradox remains for early Mars}

We conclude that a reduction in continental area and cloud fraction to yield a lower planetary albedo is not a suitable solution for the faint young Sun problem in the case of early Mars. Substantial work remains to understand the problem of early Mars climate, and climatic and geochemical models are essential tools for understanding the stability of liquid water during the Noachian. In our view, the solution will likely not come straightforward from a single standpoint, but from a combination of (i) warming from greenhouse gases in the atmosphere, whether this be $\mathrm{H}_{2} \mathrm{O}$ and $\mathrm{CO}_{2}$ alone (Kasting 1991) or in addition to some other greenhouse gases (e.g. $\mathrm{CH}_{4}, \mathrm{NH}_{3}$ ) that might have been present (Squyres \& Kasting 1994; Johnson et al. 2008), which would allow global temperatures to be close to, albeit still below, the freezing point of pure water; (ii) warming by carbon dioxide clouds (Forget \& Pierrehumbert 1997) or the transport
Mean Annual Surface Temperature for Early Mars
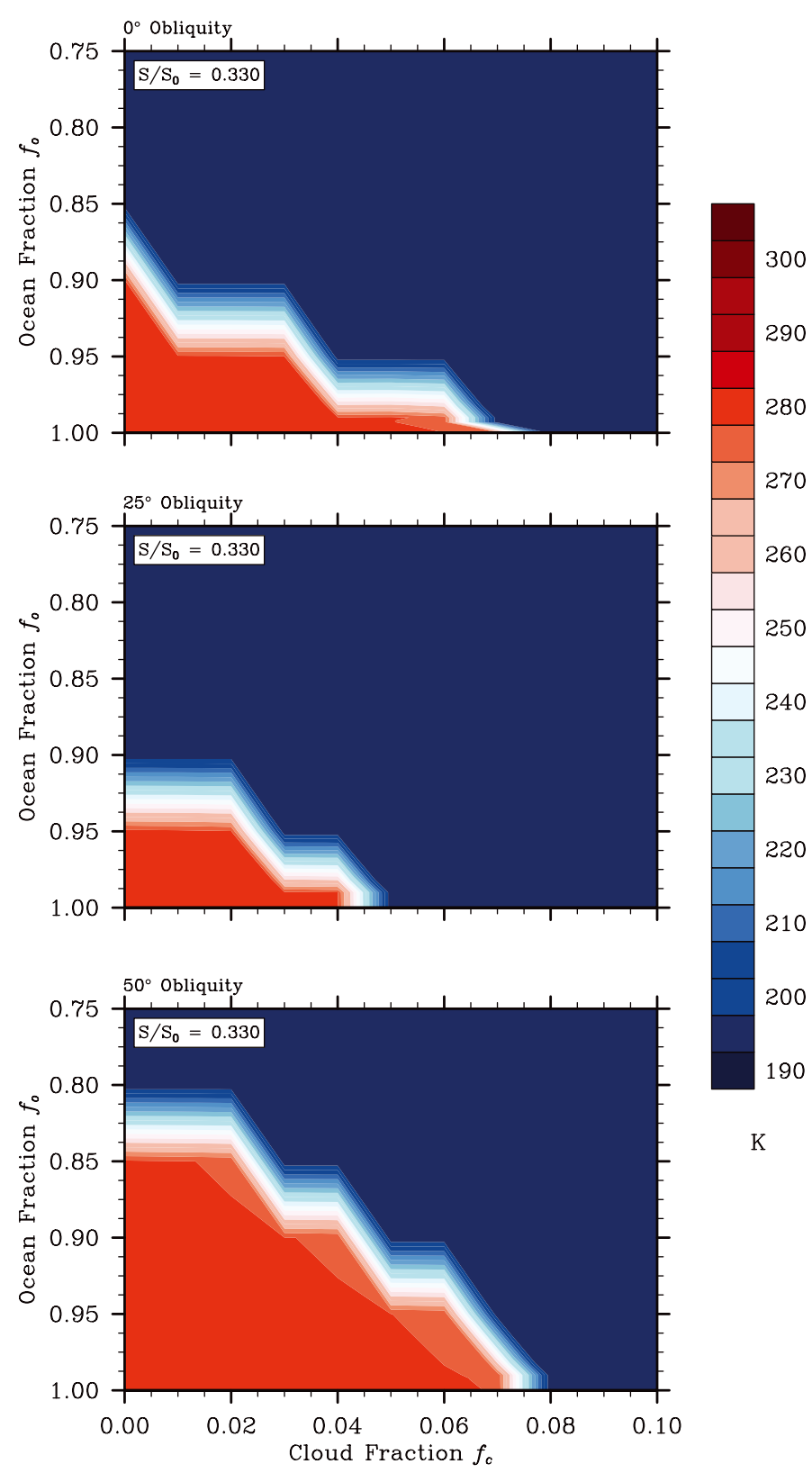

Fig. 2. Mean annual surface temperature for early Mars. Calculated at a relative solar constant $S / S_{0}=0.330$, and as a function of cloud fraction and ocean fraction. The panels show surface temperature at obliquities of $0^{\circ}$ (top), $25^{\circ}$ (middle) and $50^{\circ}$ (bottom), with a 3 bar $\mathrm{CO}_{2}-\mathrm{H}_{2} \mathrm{O}$ atmosphere.

of energy by atmospheric dynamics (Wordsworth et al. 2010), which would supplement any warming from greenhouse gases; and (iii) the geochemical properties of moderately salty solutions to depress the freezing point and allow water to remain in the liquid state on the surface of a somewhat "cold and wet" early Mars (Fairén et al. 2009; Fairén 2010).

Acknowledgements. Comments and suggestions by F. Forget have substantially contributed to improve this work.

\section{References}

Bahcall, J. N., Pinsonneault, M. H., \& Basu, S. 2001, ApJ, 555, 990 Baker, V. R., Strom, R. G., Gulick, V. C., et al. 1991, Nature, 352, 589 
Brasser, R., \& Walsh, K. J. 2011, Icarus, 213, 423

Caldeira, K., \& Kasting, J. F. 1992, Nature, 359, 226

Catling, D. C., Zahnle, K. J., \& McKay, C. P. 2001, Science, 293, 839

Clifford, S. M., \& Parker, T. J. 2001, Icarus, 154, 40

Dauphas, N., \& Kasting, J. F. 2011, Nature, 474, E1

Di Achille, G., \& Hynek, B. 2010, Nat. Geos., 3, 459

Fairén, A. G. 2010, Icarus, 208, 165

Fairén, A. G., Dohm, J. M., Baker, V. R., et al. 2003, Icarus, 165, 53

Fairén, A. G., Davila, A. F., Gago-Duport, L., et al. 2009, Nature, 459, 401

Forget, F., \& Pierrehumbert, R. T. 1997, Science, 278, 1273

Goldblatt, C., \& Zahnle, K. J. 2010, Climate of the Past Discussions, 6, 1163

Goldblatt, C., \& Zahnle, K. J. 2011, Nature, 474, E3-4

Gough, D. O. 1981, Sol. Phys., 74, 21

Grott, M., Morschhauser, A., Breuer, D., et al. 2011, Earth Planet. Sci. Lett., 308, 391

Haberle, R. M. 1998, J. Geophys. Res. Planets, 103, 28467

Halevy, I., Zuber, M. T., \& Schrag, D. P. 2007, Science, 318, 1903

Haqq-Misra, J. D., Domagal-Goldman, S. D., Kasting, P., et al. 2008, Astrobiology, 8, 1127

Head, J. W., Kreslavsky, M., Hiesinger, H., et al. 1998, Geophys. Res. Lett., 25, 4401

Irwin, R. P., Howard, A. D., Craddock, R. A., et al. 2005, J. Geophys. Res., 110, E12S15

Jacobowitz, H., Smith, W. L., Howell, H. B., et al. 1979, J. Atm. Sci., 36, 501

Johnson, S. S., Mischna, M. A., Grove, T. L., et al. 2008, J. Geophys. Res., 113, E08005

Kasting, J. F. 1991, Icarus, 94, 1

Kasting, J. F. 1993, Science, 259, 920

Kasting, J. F. 1997, Science, 276, 1213
Kasting, J. F., Pollack, J. B., \& Crisp, D. 1984, J. Atmos. Chem., 1, 403

Kiehl, J. T., \& Dickinson, R. E. 1987, J. Geophys. Res., 92(D3), 2991

Kondrat'ev, K. J. 1969, Radiation in the atmosphere (New York: Academic Press)

Kuhn, W. R., \& Atreya, S. W. 1979, Icarus, 37, 207

Laskar, J., \& Robutel, P. 1993, Nature, 361, 608

Laskar, J., Correia, A. C. M., Gastineau, M., et al. 2004, Icarus, 170, 343

Malin, M. C., \& Edgett, K. S. 2003, Science, 302, 1931

North, G. R., Cahalan, R. F., \& Coakley, Jr., J. A. 1981, Rev. Geophysics, 19, 91

North, G. R., Mengel, J. G., \& Short, D. A. 1983, J. Geophys. Res.-Oceans, 88, 6576

Pain, C. F., Clarke, J. D. A., \& Thomas, M. 2007, Icarus, 190, 478

Parker, T. J., Gorsline, D. S., Saunders, R. S., et al. 1993, J. Geophys. Res., 98, 11061

Rondanelli, R., \& Lindzen, R. S. 2010, J. Geophys. Res., 115, D02108

Rosing, M. T., Bird, D. K., Sleep, N. H., et al. 2010, Nature, 464, 744

Rosing, M. T., Bird, D. K., Sleep, N. H., et al. 2011, Reply. Nat., 474, E1

Sagan, C., \& Chyba, C. 1997, Science, 276, 1217

Sagan, C., \& Mullen, G. 1972, Science, 177, 52

Squyres, S. W., \& Kasting, J. F. 1994, Science, 265, 744

Squyres, S. W., Grotzinger, J. P., Arvidson, R. E., et al. 2004, Science, 306, 1709

Thompson, S. L., \& Barron, E. J. 1981, J. Geol., 89, 143

Tian, F., Claire, M. W., Haqq-Misra, J. D., et al. 2010, Earth Planet. Sci. Lett., 295,412

Touma, J., \& Wisdom, J. 1993, Science, 259, 1294

von Paris, P., Rauer, H., Grenfell, J. L., et al. 2008, Planet. Space Sci., 56, 1244

Williams, D. M., \& Kasting, J. F. 1997, Icarus, 129, 254

Wordsworth, R., Forget, F., \& Eymet, V. 2010, Icarus, 210, 992 